\title{
Comparison of the intensity of peripheral inflammation between major depressive disorder and bipolar depression by means of neutrophil-lymphocyte and platelet- lymphocyte ratios: The possible role of clinical severity and psychotic features
}

\author{
Necati Serkut BULUT ${ }^{1}$ (D) Nese YORGUNER ${ }^{2}$ (iD) \\ ${ }^{1}$ Department of Psychiatry, Marmara University Pendik Training and Research Hospital, Istanbul, Turkey. \\ ${ }^{2}$ Department of Psychiatry, School of Medicine, Marmara University, İstanbul, Turkey.
}

Corresponding Author: Necati Serkut BULUT

E-mail: serkutbulut@gmail.com

\begin{abstract}
Objective: The present study aimed to compare the intensity of inflammation between major depressive disorder (MDD) and bipolar disorder-depressive episode (BD-D) by using neutrophil to lymphocyte (NLR) and platelet to lymphocyte ratios (PLR) as nonspecific markers for peripheral immune response, and to investigate whether and how these parameters correlate with the clinical characteristics of the depressive episodes within and between the diagnoses.

Patients and Methods: The medical records of 209 psychiatric inpatients (126 diagnosed with MDD, 83 with BD-D) and 150 healthy controls (HC) were retrospectively screened to obtain NLR and PLR values.

Results: Both MDD and BD-D presented with significantly elevated NLR and PLR compared to HC, with the increase being associated with the severity of depression but not with the presence of psychotic features. The severity of inflammation was found to be of a comparable magnitude between the two conditions, or at least indistinguishable by means of the NLR and PLR.

Conclusion: Our results suggest that both MDD and BD-D involve a presumably complex inflammatory process resulting in an observable, albeit nonspecific alteration in the distribution of peripheric blood cells. Moreover, the magnitude of the observed immune response appears to relate to the severity of the depressive episode for both conditions.

Keywords: Major depressive disorder, Bipolar disorder, Inflammation, Immune dysregulation, Neutrophil-lymphocyte ratio, Plateletlymphocyte ratio
\end{abstract}

\section{INTRODUCTION}

Depression is extremely common in both community and treatment settings. Although depressive symptoms may occur in a variety of psychiatric and somatic conditions, depressive episodes are most specific for major depressive disorder (MDD) and bipolar disorder (BD), the two most prevalent mood disorders that affect up to one-fifth of people all around the world [1-3]. Several biological theories on the pathophysiology of depression have been proposed over the past decades (e.g., models focusing on neurotransmitters, neuroplasticity, genetics, circadian rhythm etc.). However, no single mechanism that is relevant to all types of depression has yet been identified, which underpins the complex and multi-dimensional etiology of the phenomenon. As with other complementary models, growing evidence also points out that immune dysregulation may be a crucial factor contributing to the pathophysiology of depression in both MDD and $\mathrm{BD}[4,5]$. Within this "immune-inflammatory" framework, the emphasis of the previous research has largely been put on the complex interplay between the neuroendocrine and immune systems [6]. Accordingly, it has been suggested that, during depression, the activation of the inflammatory response system together with the immune-regulatory pathways results in an increase in the levels of acute phase proteins such as haptoglobin [7], transforming growth factor (TGF)- $\beta 1$, IL- 10 , as well as the activated M1 macrophage, T helper and T regulatory cells [8-10]. Many other pro-inflammatory cytokines have also been shown to be elevated in depressed patients compared to healthy controls (HC), including C-reactive protein (CRP), soluble interleukin-2 and interleukin- 6 receptors, soluble tumor necrosis factor receptor, soluble p-selectin receptor, monocyte chemotactic protein-1, etc. $[6,11,12]$.

How to cite this article: Bulut NS, Yorguner N. Comparison of the intensity of peripheral inflammation between major depressive disorder and bipolar depression by means of neutrophil-lymphocyte and platelet-lymphocyte ratios: The possible role of clinical severity and psychotic features. Marmara Med J 2022: 35(1):100-106. doi: 10.5472/marumj.1065834 
Among this plethora of available inflammatory markers, recently described neutrophil to lymphocyte ratio (NLR) and platelet to lymphocyte ratio (PLR) stand out as relatively simple and inexpensive indices of the systemic inflammatory response. NLR has been repetitively tested as a candidate parameter for systemic inflammation in neuropsychiatric disorders such as Alzheimer's disease [13] and schizophrenia [14, 15]. A few studies also attempted to examine peripheral inflammatory response in mood disorders by means of NLR and/or PLR, with their findings collectively suggesting that MDD [16], as well as different mood episodes of BD [17] are associated with increased NLR and/or PLR compared to HC. On the other hand, some other studies addressing the relationship between clinical severity of MDD and NLR-PLR provided rather inconsistent findings $[18,19]$.

Taken together, a substantial body of evidence indicates that the elevated inflammatory signaling is a potential mechanism that is involved in the pathophysiology of depression. However, it remains largely unclear whether MDD and bipolar disorderdepressive episode (BD-D), two distinct clinical conditions with highly overlapping symptomatology, present with differential patterns of inflammatory responses. Only a handful of studies to date have attempted to investigate the potentially distinctive features of the inflammatory profiles between the two disorders, by directly comparing depressed patients diagnosed with MDD or BD-D [6, 20, 21]. Moreover, most of the available research on the relationship between inflammatory processes and mood disorders suffer from substantial limitations such as the lack of control groups, unreliability of the diagnoses (which are mostly based on the use of questionnaires), not taking into consideration the clinical features of the depressive episodes, or relying upon cross-sectional assessments based on relatively narrow DSM criteria without further corroboration.

With these methodological shortcomings taken into account, our first objective in the present study was to assess and compare the intensity of peripheral inflammation between MDD and BD-D by using NLR and PLR as non-specific markers for the systemic immune response. Our second objective was to investigate whether and how the observed values for these markers were related to the main clinical features of the depressive episodes (depression severity, presence of psychotic features) both in MDD and BD-D. To the best of our knowledge, no prior studies have directly compared the inflammatory profiles between MDD and BD-D patients by means of NLR-PLR, or by taking into consideration the main clinical characteristics of the depressive episodes.

\section{PATIENTS and METHODS}

\section{Participants}

In this cross-sectional study, the database of our psychiatry inpatient clinic has been retrospectively screened for adult patients ( $>18$ years old) who had been admitted to the hospital between 2015-2020, with a main diagnosis of MDD or BD-D. To reduce diagnostic heterogeneity, patients with psychiatric comorbidities or differential diagnoses (anxiety disorders, somatoform disorders, alcohol and substance use disorders, dissociative disorders, schizoaffective disorder, borderline personality disorder, etc.) have been excluded from the study sample. Additional exclusion criteria were the medical comorbidities that might affect inflammatory parameters (autoimmune or inflammatory diseases etc.), hepatic or renal failure, blood dyscrasia, ongoing infection, obesity (BMI $>30 \mathrm{~kg} /$ $\mathrm{m}^{2}$ ), and treatment with anti-inflammatory medications by the time of the admittance. Accordingly, the final sample consisted of a total of 209 eligible subjects, with 126 being diagnosed with MDD, and 83 with BD-D.

Aside from these two major diagnostic groups, participants have further been classified in relation to the clinical severity of their depression (moderate, severe) as well as the presence of psychotic features (additional specifier for severe depression). Additionally, a control group $(\mathrm{n}=150)$ of non-psychiatric outpatients, with no past history of psychiatric diagnosis or treatment, and similar demographic characteristics have been recruited retrospectively following the above mentioned criteria. The study protocol was approved by the Ethics Committee of Marmara University, School of Medicine (no: 09. 2019.1089).

\section{Blood Tests}

Data from the laboratory tests were obtained by retrospective screening of the medical records of the patients. As a routine, blood samples were drawn from the patients within 24 hours of their first admission to the clinic, generally around 8-10 a.m. following an overnight fasting period for at least 8 hours. Only the complete blood count (CBC) results at the time of the first admittance were taken into consideration. Accordingly, neutrophil/lymphocyte and platelet/lymphocyte ratios were calculated for each patient, using absolute counts.

\section{Statistical Analysis}

Data analysis in the present work was performed using SPSS (version 24.0). Means (M), standard deviation (SD) and percentages $(\%)$ were used to illustrate the demographic and other selected characteristics of the participants. KolmogorovSmirnov and Shapiro-Wilk were used to determine normal distribution. The chi-square test was used for categorical variables. Student's t-test or the Mann-Whitney U test were used to compare ordinal variables between two independent groups, Kruskal-Wallis or the one-way ANOVA were used to compare three or more groups. Analysis of covariance (ANCOVA) was used to control for covariates of interest when looking at group differences. A two-tailed $\mathrm{p}<0.05$ was considered statistically significant.

\section{RESULTS}

\section{Sample Characteristics}

The sample consisted of 359 participants in total (Table I). $65.1 \%(n=82)$ of the MDD, $72.3 \%(n=60)$ of the BD-D, and $58 \%(n=87)$ of the HC were females, the gender distribution 
between groups was not significantly different $(\mathrm{p}=0.088)$. The mean age was $46.06(\mathrm{SD}=17.39)$ for $\mathrm{MDD}, 40.59(\mathrm{SD}=13.76)$ for $\mathrm{BD}-\mathrm{D}$, and $44.18(\mathrm{SD}=14.83)$ for $\mathrm{HC}$. The age difference between groups was not statistically significant $(\mathrm{p}>0.05)$. The depression severity was moderate in $48.4 \%(n=61)$ of the MDD and $50.6 \%(n=42)$ of the BD-D patients. Among those with severe depression $(n=106), 40 \%$ of the MDD $(n=26)$ and $24.3 \%$ $(n=10)$ of the BD-D additionally exhibited psychotic features during the episode. The distribution of the patients by symptom severity $(\chi 2=0.096, p=0.757)$, and presence of psychotic features $(\chi 2=2.73, \mathrm{p}=0.098)$ were similar in $\mathrm{MDD}$ and $\mathrm{BD}-\mathrm{D}$ groups, i.e. without statistically significant difference. Regardless of the main diagnosis, $54.9 \%(n=78)$ of the female and $41.7 \%(n=28)$ of the male patients had severe depression, among which $33.3 \%$ $(n=26)$ and $35.7 \%(n=10)$ presented with additional psychotic features respectively. The mean duration of hospital stay was comparable (i.e., without statistically significant difference) between MDD and BD-D, with $5.45(\mathrm{SD}=2.97)$ and 5.67 $(\mathrm{SD}=2.87)$ weeks respectively $(\mathrm{p}>0.05)$.

Table I. Sample characteristics

\begin{tabular}{|c|c|c|c|c|c|c|c|c|c|}
\hline \multirow[t]{2}{*}{ Groups } & \multirow[t]{2}{*}{$\begin{array}{l}\text { Symptom } \\
\text { Severity }\end{array}$} & \multirow[t]{2}{*}{$\begin{array}{l}\text { Clinical } \\
\text { Features }\end{array}$} & \multicolumn{3}{|c|}{ Gender (n, \%) } & \multicolumn{2}{|c|}{ Age (Mean \pm SD) } & \multicolumn{2}{|c|}{$\begin{array}{l}\text { Duration of Hospitalization } \\
(\text { Mean } \pm \text { SD) }\end{array}$} \\
\hline & & & Female & Male & Total & Subgroups & Groups & Subgroups & Groups \\
\hline Healthy Controls & & & $87(58 \%)$ & $63(42 \%)$ & 150 & & $44.18 \pm 14.83$ & & \\
\hline \multirow{3}{*}{ Major Depressive Disorder } & Moderate & & $35(57.4 \%)$ & $26(42.6 \%)$ & 61 & $41.62 \pm 15.86$ & \multirow{3}{*}{$46.06 \pm 17.39$} & $4.79 \pm 2.74$ & \multirow{3}{*}{$5.45 \pm 2.97$} \\
\hline & Severe & non-psychotic & $29(74.4 \%)$ & $10(25.6 \%)$ & 39 & $48.23 \pm 19.45$ & & $5.98 \pm 2.67$ & \\
\hline & & psychotic & $18(69.2 \%)$ & $8(30.8 \%)$ & 26 & $53.19 \pm 14.98$ & & $6.19 \pm 3.62$ & \\
\hline \multirow{3}{*}{ Bipolar Depression } & Moderate & & $29(69 \%)$ & $13(31 \%)$ & 42 & $37.90 \pm 12.99$ & \multirow{3}{*}{$40.59 \pm 13.76$} & $5.62 \pm 2.79$ & \multirow{3}{*}{$5.67 \pm 2.87$} \\
\hline & Severe & non-psychotic & $23(74.2 \%)$ & $8(25.8 \%)$ & 31 & $43.39 \pm 13.38$ & & $5.59 \pm 3.26$ & \\
\hline & & psychotic & $8(80 \%)$ & $2(20 \%)$ & 10 & $43.20 \pm 17.04$ & & $6.16 \pm 2.03$ & \\
\hline TOTAL & & & 229 & 130 & 359 & & & & \\
\hline
\end{tabular}

\section{Comparison between the diagnostic groups}

Mean NLR values were 1.59 (SD=0.74) for $\mathrm{HC}, 2.30(\mathrm{SD}=1.51)$ for $\mathrm{MDD}$, and $2.32(\mathrm{SD}=0.97)$ for $\mathrm{BD}-\mathrm{D}$ group. Mean PLR values were $110.88(\mathrm{SD}=37.71)$ for $\mathrm{HC}, 135.29(\mathrm{SD}=62.92)$ for $\mathrm{MDD}$, and 122.33 ( $\mathrm{SD}=51.93)$ for $\mathrm{BD}-\mathrm{D}$ group. Raw values were logtransformed using natural logarithm to obtain normal distribution (Table II). Log-transformed values are denoted with the prefix Ln.

Both LnNLR and LnPLR values exhibited a significant difference between groups even after adjusting for age and sex with
$\operatorname{ANCOVA}(\mathrm{F}=24.69, \mathrm{p}<0.001$; and $\mathrm{F}=6.02, \mathrm{p}=0.003$ respectively) (Table III). Post-hoc analyses revealed that, both MDD and BD-D presented with significantly higher LnNLR values, compared to controls ( $\mathrm{p}<0.001$ for both), while no significant difference was observed between MDD and BD-D ( $\mathrm{p}=0.554)$. Regarding LnPLR values, MDD was no different from BD-D ( $\mathrm{p}=0.084)$ but significantly higher than $\mathrm{HC}(\mathrm{p}=0.001)$, whereas the difference between BD-D and HC was not significant $(p=0.331)$.

Table II. Mean LnNLR and LnPLR in relation to the clinical characteristics among diagnostic groups and healthy controls

\begin{tabular}{|c|c|c|c|c|c|c|c|c|c|c|c|}
\hline \multirow{3}{*}{ 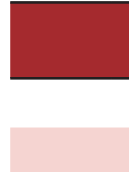 } & \multirow[t]{3}{*}{$\begin{array}{l}\text { Healthy } \\
\text { Controls }\end{array}$} & \multicolumn{5}{|c|}{ Major Depressive Disorder } & \multicolumn{5}{|c|}{ Bipolar Depression } \\
\hline & & Moderate & & Severe & & Overall & Moderate & & evere & & Overall \\
\hline & & & Non-psychotic & Psychotic & Overall & & & Non-psychotic & Psychotic & Overall & \\
\hline LnNLR & $0.37 \pm 0.42$ & $0.63 \pm 0.40$ & $0.80 \pm 0.54$ & $0.70 \pm 0.55$ & $0.76 \pm 0.54$ & $0.70 \pm 0.48$ & $0.65 \pm 0.38$ & $0.89 \pm 0.44$ & $0.74 \pm 0.44$ & $0.85 \pm 0.43$ & $0.75 \pm 0.42$ \\
\hline LnPLR & $4.65 \pm 0.31$ & $4.73 \pm 0.39$ & $4.89 \pm 0.37$ & $4.90 \pm 0.45$ & $4.90 \pm 0.40$ & $4.82 \pm 0.40$ & $4.68 \pm 0.39$ & $4.80 \pm 0.40$ & $4.75 \pm 0.29$ & $4.79 \pm 0.37$ & $4.74 \pm 0.39$ \\
\hline
\end{tabular}

Ln: natural logarithm, NLR: neutrophil to lymphocyte ratio, PLR: platelet to lymphocyte ratio

\section{Comparison between the depressive episodes in relation to clinical characteristics}

Regardless of the diagnostic groups (all patients with depression), mean $\mathrm{LnNLR}$ values were $0.64(\mathrm{SD}=0.39)$ for moderate and $0.80(\mathrm{SD}=0.50)$ for severe depression with the difference being statistically significant $(\mathrm{t}=-2.48, \mathrm{p}=0.014)$. Similarly, LnPLR showed significant difference between groups with the mean values being $4.71(\mathrm{SD}=0.39)$ for moderate, and $4.85(\mathrm{SD}=0.39)$ for severe depression $(\mathrm{t}=-2.65, \mathrm{p}=0.009)$. However, among patients with severe depression, both NLR and PLR presented with comparable values (i.e., not statistically significant) between those with and without psychotic symptoms $(\mathrm{t}=1.21$, $\mathrm{p}=0.226$, and $\mathrm{t}=-0.130, \mathrm{p}=0.897$ respectively). 
Table III. Comparison of LnNLR and LnPLR in relation to clinical characteristics between the diagnostic groups

\begin{tabular}{|c|c|c|c|c|}
\hline & \multicolumn{2}{|c|}{ LnNLR } & \multicolumn{2}{|c|}{ LnPLR } \\
\hline Comparison & Test statistic & p value & Test statistic & p value \\
\hline Difference between $H C, M D D, B D-D^{*}$ & $F=24.69$ & $<0.001$ & $F=6.02$ & 0.003 \\
\hline$B D-D$ vs $H C$ & & $<0.001$ & & 0.331 \\
\hline$M D D$ vs $H C$ & & $<0.001$ & & 0.001 \\
\hline$M D D$ vs $B D-D$ & & 0.554 & & 0.084 \\
\hline Moderate vs severe depression (overall)** & $t=-2.48$ & 0.014 & $t=-2.65$ & 0.009 \\
\hline Non-psychotic vs psychotic severe depression (overall) ${ }^{* *}$ & $t=1.21$ & 0.226 & $t=-0.130$ & 0.897 \\
\hline$M D D$, moderate vs severe ${ }^{* *}$ & $t=-1.52$ & 0.129 & $t=-2.34$ & 0.020 \\
\hline$B D-D$, moderate vs severe ${ }^{* *}$ & $t=-2.19$ & 0.031 & $t=-1.26$ & 0.211 \\
\hline
\end{tabular}

Ln: natural logarithm, NLR: neutrophil to lymphocyte ratio, PLR: platelet to lymphocyte ratio HC: healthy controls, MDD: major depressive disorder, BD-D: bipolar disorder-depressive episode

${ }^{*}$ Analysis of covariance (ANCOVA), ${ }^{*}$ Student's $t$ test

\section{Comparison between the diagnostic groups in relation to clinical characteristics}

LnNLR and LnPLR values in relation to the depression severity and the presence of psychotic symptoms for both the MDD and BD-D groups are shown in Figure 1 and Figure 2 respectively.

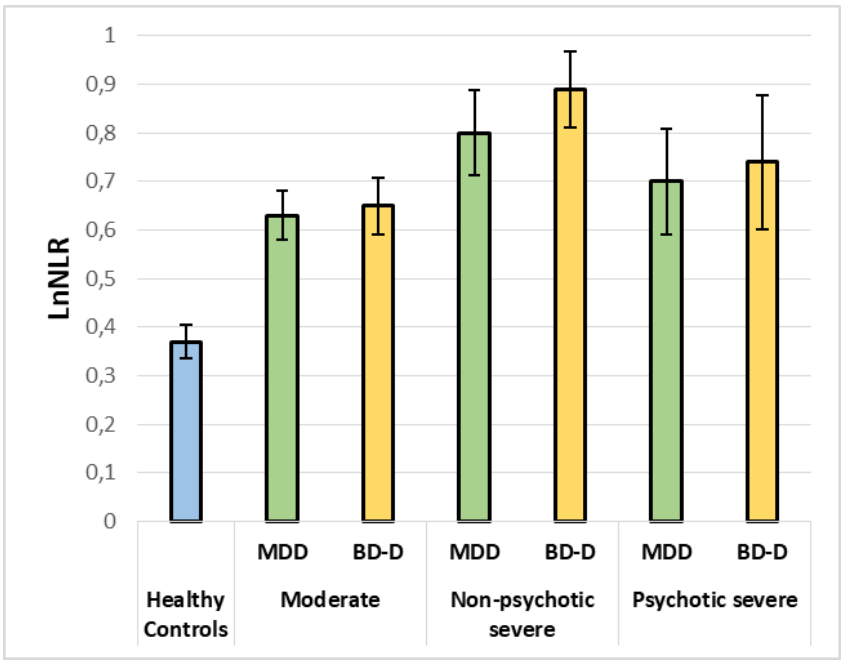

Figure 1. Mean LnNLR values in relation to the clinical characteristics for healthy controls, patients with major depressive disorder, and bipolar depression. Bars indicate standard errors. Ln: natural logarithm, NLR: neutrophil to lymphocyte ratio, $M D D$ : major depressive disorder, $B D-D$ : bipolar disorder-depressive episode

LnNLR in moderate, severe (non-psychotic) and severe psychotic depression was $0.63(\mathrm{SD}=0.40), 0.80 \quad(\mathrm{SD}=0.54)$ and 0.70 $(\mathrm{SD}=0.55)$ in the MDD; and $0.65(\mathrm{SD}=0.38), 0.89(\mathrm{SD}=0.44)$ and $0.74(\mathrm{SD}=0.44)$ in the $\mathrm{BD}-\mathrm{D}$ group respectively. That is, severe depression without psychotic features were associated with the highest, and moderate depression with the lowest LnNLR values in both groups. Although the difference between moderate and non-psychotic severe depression was found to be significant in the BD-D ( $\mathrm{p}=0.026)$, it did not reach statistical significance in the MDD group $(\mathrm{p}=0.87)$. With the psychotic features not taken into account, severe depression was again associated with significantly higher LnNLR than moderate depression for BD-D $(\mathrm{p}=0.031)$, but not for MDD $(\mathrm{p}=0.129)$.

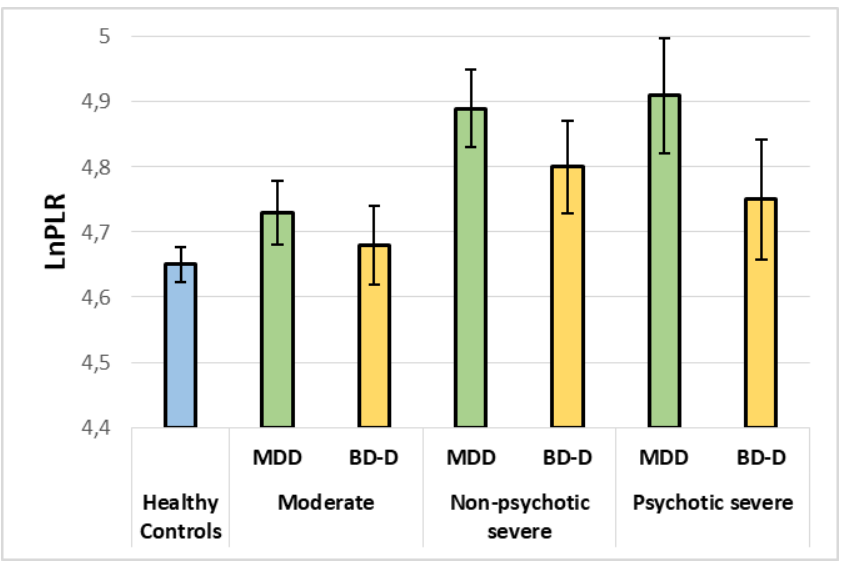

Figure 2. Mean LnNLR values in relation to the clinical characteristics for healthy controls, patients with major depressive disorder, and bipolar depression. Bars indicate standard errors. Ln: natural logarithm, PLR: platelet to lymphocyte ratio, $M D D$ : major depressive disorder, $B D-D$ : bipolar disorder-depressive episode

LnPLR in moderate, severe (non-psychotic) and severe psychotic depression was $4.73(\mathrm{SD}=0.39), 4.89(\mathrm{SD}=0.37)$ and 4.91 $(\mathrm{SD}=0.45)$ in the MDD; and $4.68(\mathrm{SD}=0.39), 4.80(\mathrm{SD}=0.40)$ and $4.75(\mathrm{SD}=0.29)$ in the $\mathrm{BD}-\mathrm{D}$ group respectively. In contrast with LnNLR, LnPLR significantly distinguished between moderate and severe depression in the MDD $(p=0.020)$, but not in the BD-D ( $\mathrm{p}=0.21)$.

\section{DISCUSSION}

In the present study, it was aimed to compare between the intensity of the accompanying inflammation in MDD and BD-D by using NLR and PLR as non-specific indices of the peripheral immune response; and to investigate whether and how these parameters correlate with the clinical features of depression 
within and between diagnoses. Our findings showed that the two disorders presented with largely similar profiles in terms of NLR and PLR, with strikingly close mean values between the diagnoses. On the other hand, both indices presented with significantly increased values in depressed patients compared to HC, supporting the ever-growing evidence on the involvement of the inflammatory mechanisms in the pathophysiology of depression.

Although, previous research indicates that both disorders are associated with altered NLR and/or PLR [16], findings regarding the potential differences between the inflammatory profiles of MDD and BD-D are rather inconsistent. Hung et al., compared the levels of CRP, TNF-alpha, and IL-6 in a sample of patients with reactive depression, MDD, BD-D and healthy controls. Their findings revealed no statistically significant difference among the groups [22]. Similarly, Su et al., reported no significant difference in CRP, soluble TNF-R1, or IL-6 levels, among patients with reactive depression, MDD, bipolar depression, and healthy controls [23]. In their retrospective cross-sectional study, Wysokinski et al., compared CRP levels between inpatients diagnosed with schizophrenia, bipolar mania, BD-D and MD-D. Again, the authors failed to find any difference in CRP levels between the disorders [24].

On the other hand, Bai et al., compared inflammatory profiles between outpatients diagnosed with $\mathrm{BD}, \mathrm{MD}-\mathrm{D}$ and $\mathrm{HC}$ by means of a series of pro-inflammatory cytokines, and found that inflammatory dysregulation was more intense in BD [6]. Mao et al., attempted to explore the difference in pro-inflammatory and anti-inflammatory cytokines between MDD and BD-D by conducting a longitudinal study on a sample of outpatients with MDD ( $n=64), B D-D(n=61)$ and healthy controls $(n=62)$. Their findings indicated that MDD and BD-D patients present with dissimilar inflammatory profiles, in terms of TNF- $\alpha$ and IL-13 levels in particular [25]. Finally, Brunoni et al., examined differences in immune profiles among MDD $(n=245)$ and BD-D $(n=59)$ patients who had depressive episode of moderate severity. The authors concluded that differences in immune profiles between BD-D and MDD patients do exist, with increased IL-10 being the primary immune-regulatory mechanism for MDD, and increased sTNFR2 and KLOTHO being the primary regulatory mechanisms for $\mathrm{BD}-\mathrm{D}$ [9].

In partial contrast with these latter reports, our results pointed out that whatever the underlying immune mechanisms, the intensity of the resulting peripheral inflammation was not different between the two disorders, or at least undistinguishable by means of the NLR or PLR. On the other hand, the severity of the depression appeared to be associated with the magnitude of the increase in NLR and/or PLR in both disorders. That is, higher values were recorded for severe depression compared to moderate in both MDD and BD-D. Of note, this pattern was inconsistently reflected by the two parameters between the disorders, so that the difference was significant for NLR but not for PLR in BD-D group, whereas the opposite was true for MDD. However, the methodological framework of our study does not allow us to determine whether or not this observed relation was due to a true difference concerning diagnostic specificity between the two markers. Another striking finding of our study was that the presence of psychotic features did not seem to have any additional impact on the increase in NLR or PLR among severely depressed patients, implying that the intensity of peripheral inflammation is more closely related to depression severity than accompanying psychotic symptomatology.

Limited literature provides contradicting evidence concerning the relationship between clinical features of the depression and NLR-PLR indices. In their study conducted on MDD patients, Kayhan et al., reported higher levels of PLR in participants who had severe depression with psychotic features, compared to those with other types of depression, although this difference was not reflected in NLR [18]. In contrast, Sunbul et al., found that higher HAM-D scores in MDD patients was associated with larger increases in NLR [19]. Similarly, CRP levels and NLR were reported to be significantly higher in MDD patients with a recent history of suicide attempt compared to those without [26].

\section{Limitations and Strengths}

The present findings should be interpreted cautiously, within the methodological limitations of our study. The retrospective screening precluded us from considering several confounding factors that might have played a role in the mechanism of interaction between depression and inflammatory processes in our sample. These missing data include the exact BMI values for each patient, smoking status, age of onset of the mood disorder, number of episodes, and family history. The potential effect of the ongoing drug treatments was also discarded due to the methodological complexity related to polypharmacy; besides, only a minority of patients were drug-free at the time of admittance.

We also believe that our study has some substantial strengths to underline. First, unlike most previous studies, a high level of diagnostic accuracy was suggested to be established among the participants. The main reason for this is that, the sample consisting solely of inpatients in our clinic, the clinical diagnoses were based not on cross-sectional assessments but longitudinal and elaborate psychiatric evaluation, supported by standardized questionnaires, medical examination and comprehensive laboratory work-up. Moreover, the final diagnoses were confirmed by a thorough retrospective examination of the patients' medical records. In doing so, the inherent difficulty to cross-sectionally differentiate between unipolar and bipolar depression was suggested to have been largely overcome. Finally, we believe that the exclusion of depressed patients with psychiatric comorbidities/differential diagnoses, as well as the identification of the main clinical features of the depressive episodes have provided the possibility to delineate relatively well distinguished sub-groups with increased homogeneity.

\section{Conclusion}

Taken together, our results suggest that the pathophysiology of both BD-D and MDD involves presumably complex inflammatory processes which result in an observable -albeit nonspecific - alteration in the distribution of peripheric blood 
cells. The depressive episodes in both disorders present with significantly increased levels of NLR and PLR compared to $\mathrm{HC}$, with greater values being additionally associated with severe depression, but not necessarily with the presence of psychotic features. Finally, the intensity of the accompanying inflammation appears to be comparable between the disorders, or at least, indistinguishable by means of the NLR and PLR. Given the substantial symptomatic overlap and high rate of misdiagnosis between the two disorders, researchers addressing the differential characteristics of inflammation between MDD and BD-D should not content themselves with the use of a wider range of specific bio-markers but also attempt to adopt more elaborate sampling methodologies, e.g. by enhancing diagnostic accuracy among participants through multi-dimensional clinical assessments, as well as addressing the clinical features of the depression with potential relevance (bipolar subtypes, cycling rates, family history, presence of melancholic, atypical or mixed features, etc.).

It is without a doubt that the study of inflammatory mechanisms will continue to enrich our understanding of the pathophysiology of depression. However, ranging from basic to the most sophisticated laboratory tests, biological markers are yet to be adopted as reliable diagnostic tools to differentiate between MDD and BD-D. As in the other fields of psychiatry, clinical expertise and elaborate psychiatric assessment remain the keystone of an accurate diagnosis.

\section{Compliance with Ethical Standards}

Ethical Approval: Approval for the study was obtained from the Ethics Committee of Marmara University, School of Medicine with the protocol number 09.2019.1089.

Financial Support: The authors have no relevant financial information to disclose.

Declaration of Competing Conflict of Interest: The authors declare that there are no conflicts of interest.

Author contributions: Both authors were actively involved in data collection, analysis, and the writing of the article.

\section{REFERENCES}

[1] Leonpacher A, Liebers D, Pirooznia M, et al. Distinguishing bipolar from unipolar depression: the importance of clinical symptoms and illness features. Psychol Med 2015;45:2437-46. doi: 10.1017/S003.329.1715000446.

[2] Bromet E, Andrade LH, Hwang I, et al. Cross-national epidemiology of DSM-IV major depressive episode. BMC Med 2011;9:90. doi: 10.1186/1741-7015-9-90.

[3] Merikangas KR, Jin R, He J-P, et al. Prevalence and correlates of bipolar spectrum disorder in the world mental health survey initiative. Arch Gen Psychiatry 2011;68:241-51. doi: 10.1001/archgenpsychiatry.2011.12.

[4] Gibney SM, Drexhage HA. Evidence for a dysregulated immune system in the etiology of psychiatric disorders. J Neuroimmune Pharmacol 2013;8:900-20. doi: 10.1007/ s11481.013.9462-8.
[5] Vogelzangs N, Duivis HE, Beekman AT, et al. Association of depressive disorders, depression characteristics and antidepressant medication with inflammation. Transl Psychiatry 2012;2:e79-e79. doi: 10.1038/tp.2012.8.

[6] Bai YM, Su TP, Li CT, et al. Comparison of pro-inflammatory cytokines among patients with bipolar disorder and unipolar depression and normal controls. Bipolar Disorders 2015;17:269-77.

[7] Köhler C, Freitas T, Maes Md, et al. Peripheral cytokine and chemokine alterations in depression: a meta-analysis of 82 studies. Acta Psychiatr Scand 2017;135:373-87. doi: 10.1111/ acps. 12698 .

[8] Maes M, Carvalho AF. The compensatory immune-regulatory reflex system (CIRS) in depression and bipolar disorder. Mol Neurobiol 2018;55:8885-8903 doi: 10.1007/s12035.018.1016-x.

[9] Brunoni AR, Supasitthumrong T, Teixeira AL, et al. Differences in the immune-inflammatory profiles of unipolar and bipolar depression. J Affect Disord 2020;262:8-15. doi: 10.1016/j. jad.2019.10.037.

[10] Wang Q, Yu C, Shi S, et al. An analysis of plasma reveals proteins in the acute phase response pathway to be candidate diagnostic biomarkers for depression. Psychiatry Res 2019;272:404-10. doi: 10.1016/j.psychres.2018.11.069.

[11] Maes M, Bonifacio KL, Morelli NR, et al. Major differences in neurooxidative and neuronitrosative stress pathways between major depressive disorder and types I and II bipolar disorder. Mol Neurobiol 2019;56:141-56. doi: 10.1007/s12035.018.10517.

[12] Al-Hakeim HK, Al-Rammahi DA, Al-Dujaili AH. IL6, IL-18, sIL-2R, and TNFa proinflammatory markers in depression and schizophrenia patients who are free of overt inflammation. J Affect Disord 2015;182:106-14. doi: 10.1016/j. jad.2015.04.044.

[13] Kuyumcu ME, Yesil Y, Oztürk ZA, et al. The evaluation of neutrophil-lymphocyte ratio in Alzheimer's disease. Dement Geriatr Cogn Disord 2012;34:69-74. doi: 10.1159/000341583.

[14] Semiz M, Yildirim O, Canan F, et al. Elevated neutrophil/ lymphocyte ratio in patients with schizophrenia. Psychiatr Danub 2014;26:220-5.

[15] Kulaksizoglu B, Kulaksizoglu S. Relationship between neutrophil/lymphocyte ratio with oxidative stress and psychopathology in patients with schizophrenia. Neuropsychiatr Dis Treat 2016;12:1999. doi: 10.2147/NDT. S110484.

[16] Demir S, Atli A, Bulut M, et al. Neutrophil-lymphocyte ratio in patients with major depressive disorder undergoing no pharmacological therapy. Neuropsychiatr Dis Treat 2015;11:2253. doi: 10.2147/NDT.S89470.

[17] Giynas Ayhan M, Cicek IE, Inanli I, et al. Neutrophil/ lymphocyte and platelet/lymphocyte ratios in all mood states of bipolar disorder. Psychiatr Clin Psychopharmacol 2017;27:278-82. doi: 10.1080/24750.573.2017.1338822.

[18] Kayhan F, Gündüz Ş, Ersoy SA, et al. Relationships of neutrophil-lymphocyte and platelet-lymphocyte ratios with 
the severity of major depression. Psychiatry Res 2017;247:3325. doi: doi.org/10.1016/j.psychres.2016.11.016.

[19] Sunbul EA, Sunbul M, Yanartas O, et al. Increased neutrophil/ lymphocyte ratio in patients with depression is correlated with the severity of depression and cardiovascular risk factors. Psychiatry Investig 2016;13:121. doi: 10.4306/pi.2016.13.1.121.

[20] Chang HH, Chen PS. C-reactive protein as a differential biomarker of bipolar versus unipolar depression: Response. World J Biol Psychiatry 2017;18:73-4. doi: 10.1080/15622.975.2016.1208845.

[21] Mota R, Gazal M, Acosta BA, et al. Interleukin- $1 \beta$ is associated with depressive episode in major depression but not in bipolar disorder. J Psychiatr Res 2013;47:2011-4. doi: 10.1016/j. jpsychires.2013.08.020.

[22] Hung YJ, Hsieh $\mathrm{CH}$, Chen YJ, et al. Insulin sensitivity, proinflammatory markers and adiponectin in young males with different subtypes of depressive disorder. Clin Endocrinol 2007;67:784-9. doi: 10.1111/j.1365-2265.2007.02963.x.
[23] Su SC, Sun MT, Wen MJ, et al. Brain-derived neurotrophic factor, adiponectin, and proinflammatory markers in various subtypes of depression in young men. Int J Psychiatry Med 2011;42:211-26. doi: 10.2190/PM.42.3.a.

[24] Wysokiński A, Margulska A, Strzelecki D, et al. Levels of C-reactive protein (CRP) in patients with schizophrenia, unipolar depression and bipolar disorder. Nord J Psychiatry 2015;69:346-53. doi: 10.3109/08039.488.2014.984755.

[25] Mao R, Zhang C, Chen J, et al. Different levels of pro-and antiinflammatory cytokines in patients with unipolar and bipolar depression. J Affect Disord 2018;237:65-72. doi: 10.1016/j. jad.2018.04.115.

[26] Ekinci O, Ekinci A. The connections among suicidal behavior, lipid profile and low-grade inflammation in patients with major depressive disorder: a specific relationship with the neutrophil-to-lymphocyte ratio. Nord J Psychiatry 2017;71:574-80. doi: 10.1080/08039.488.2017.1363285. 